\title{
Estrategias de control y mitigación de contagio por COVID-19 en un hospital psiquiátrico en México
}

\author{
Guillermo León-González, ${ }^{1}$ Odón A. Pavón-Gutiérrez, ${ }^{2}$ Carlos Téllez-Santillán, ${ }^{3}$ \\ Francisca Sánchez-Carmona ${ }^{4}$ y Karina Yáñez-Castañeda * \\ 'Dirección; ${ }^{2}$ Subdirección; ${ }^{3}$ Subdirección Médica; ${ }^{4}$ Departamento de Psicología. Hospital Psiquiátrico "José Sayago", Estado de México, México
}

\section{Resumen}

En el contexto de la emergente pandemia de COVID-19, uno de los grandes desafíos es generar estrategias eficaces de control de infecciones nosocomiales, específicamente en hospitales psiquiátricos con población considerada de riesgo (adultos mayores o con comorbilidades). En el presente artículo se describen las estrategias de prevención, contención y tratamiento de contagio, a partir de un brote de COVID-19 ocurrido en julio de 2020 en un hospital psiquiátrico del Estado de México. La población estuvo constituida por mujeres con estancia hospitalaria prolongada (media = 24 años), en su mayoría geriátricas (media = 64 años), con trastornos psiquiátricos diversos y comorbilidades. En total se diagnosticaron 19 casos positivos de COVID-19, de los cuales 13 cursaron con sintomatología leve y seis resultaron asintomáticos. No se presentaron alteraciones en el estado mental, en la sintomatología psiquiátrica ni en las enfermedades de base. Se realizaron algoritmos para el manejo y tratamiento de los casos sospechosos o confirmados de COVID-19. Finalmente, se consideró que la generación de estrategias integrales, acciones rápidas y oportunas, así como una adecuada gestión de recursos humanos favorecedora del trabajo interdisciplinario contribuyeron a contener y mitigar el brote de COVID-19, constituyéndose en un precedente en el ámbito psiquiátrico con pacientes institucionalizadas.

PALABRAS CLAVE: COVID-19. Hospital psiquiátrico. Prevención y control. Trastornos mentales.

\section{Strategies for COVID-19 transmission control and mitigation in a psychiatric hospital in Mexico}

\begin{abstract}
In the context of the emerging COVID-19 pandemic, one of the great challenges is to generate effective strategies for the control of nosocomial infections, specifically in psychiatric hospitals with populations considered at risk (older adults or individuals with comorbidities). This article describes the strategies for prevention, containment and treatment of infection transmission implemented during a COVID-19 outbreak that occurred in July 2020 in a psychiatric hospital of the State of Mexico. The population was comprised by women with prolonged hospital stay (mean $=24$ years), mostly geriatric (mean $=64$ years), with various psychiatric disorders and comorbidities. In total, 19 COVID-19-positive cases were diagnosed, out of which thirteen had mild symptoms and six were asymptomatic. There were no alterations in mental state, psychiatric symptoms or underlying diseases. Algorithms were developed for the management and treatment of suspected/confirmed COVID-19 cases. Finally, the generation of comprehensive strategies, quick and timely actions, as well as adequate management of human resources favoring interdisciplinary work, were deemed to have contributed to contain and mitigate the COVID-19 outbreak, which constitutes a precedent in the psychiatric field with institutionalized patients.
\end{abstract}

KEY WORDS: COVID-19. Psychiatric hospital. Prevention and control. Mental disorders.

Correspondencia:

*Karina Yáñez-Castañeda

E-mail: unidaddeinvestigacionhpjs@gmail.com
Fecha de recepción: 13-11-2020

Fecha de aceptación: 10-12-2020

DOI: $10.24875 / G M M .20000824$
Gac Med Mex. 2021;157:459-463

Disponible en PubMed

www.gacetamedicademexico.com

0016-3813/@ 2021 Academia Nacional de Medicina de México, A.C. Publicado por Permanyer. Este es un artículo open access bajo la licencia CC BY-NC-ND (http://creativecommons.org/licenses/by-nc-nd/4.0/). 


\section{Introducción}

La enfermedad por COVID-19 fue identificada primeramente como un brote de enfermedad respiratoria en la ciudad de Wuhan, provincia de Hubei, China. ${ }^{1}$ Dada la expansión de la enfermedad, el 30 de enero de 2020, la Organización Mundial de la Salud (OMS) declaró una emergencia sanitaria global y el 11 de marzo del mismo año declaró a COVID-19 como pandemia.

En este contexto, contener la transmisión de infecciones en ambientes hospitalarios ha resultado todo un reto, especialmente en hospitales psiquiátricos, debido al deterioro cognitivo de los pacientes, aunado a la poca conciencia de riesgo y la estancia prolongada. ${ }^{2}$ En adición, la presencia de enfermedades crónicas precedentes incrementa las posibilidades de presentar cuadros más graves, ${ }^{3,4}$ específicamente diabetes mellitus, hipertensión arterial sistémica y enfermedad pulmonar obstructiva crónica. ${ }^{5,6}$

Lo emergente de esta pandemia obstaculizó que se reaccionara oportunamente ante la propagación del virus, por lo que de inmediato se presentaron brotes de COVID-19 en hospitales psiquiátricos, ${ }^{7}$ donde los principales factores de riesgo de infección nosocomial constituyeron la saturación de las salas y la poca respuesta de los pacientes debido a su condición mental. ${ }^{8}$ Lo anterior ha hecho evidente la existencia de un vacío en los protocolos de respuesta ante epidemias, por lo que se recomienda aprender de la experiencia previa ${ }^{9}$ en otros países, en los cuales se han reportado las medidas implementadas ante brotes epidemiológicos con el fin de evitar infecciones nosocomiales subsecuentes. ${ }^{10}$

En México, las instituciones gubernamentales ordenaron medidas encaminadas a la prevención y mitigación de COVID-19 en hospitales psiquiátricos. ${ }^{11}$ Sin embargo, se han reportado casos positivos de la enfermedad que incluso han derivado en muertes.12 La generalidad de los lineamientos gubernamentales obliga a realizar adecuaciones según las necesidades del tipo de población que reside en cada hospital, por lo que el objetivo de este artículo es describir las estrategias de prevención, contención y tratamiento de contagio, a partir de la ocurrencia de un brote de COVID-19 en un hospital psiquiátrico público ubicado en el Estado de México, en el que residen 171 mujeres con trastornos mentales diversos, con edad de 21 a 99 años (aunque en la mayoría fue superior a 60 años). Las usuarias cohabitan en "villas", espacios

\section{Estrategias de prevención}

- Priorización de la detección de síntomas

- Establecimiento de filtro sanitario para el acceso al hospital

- Suspensión temporal del servicio de consulta externa

- Suspensión temporal de visitas familiares y domiciliarias

- Establecimiento de programas de capacitación virtual en torno al tema COVID-19

- Suministro de equipo de protección personal

- Resguardo del personal que presente síntomas sospechosos de COVID-19

- Apertura del servicio de telemedicina

- Fomento de medidas de autocuidado y sana distancia

- Establecimiento de horarios alternados en el servicio de comedor

y su adecuación espacial con el objetivo de mantener la sana

distancia

- Establecimiento de labores escalonadas en áreas de servicio esenciales

- Realización de tamizaje (mediante la prueba RT-PCR)

- Elaboración de algoritmo para el manejo de pacientes

sospechosos o confirmados con COVID-19

- Elaboración de algoritmo para el tratamiento de pacientes

sospechosos o confirmados con COVID-19

Figura 1. Estrategias de prevención implementadas en un hospital psiquiátrico ante el brote de COVID-19.

destinados a 12 usuarias en promedio y que incluye cuatro dormitorios, un sanitario por dormitorio, un área común, un comedor y el área de control médico.

\section{Medidas de prevención}

Al respecto, se realizaron adecuaciones a los lineamientos que dispuso la Secretaría de Salud en coordinación con los Servicios de Atención Psiquiátrica y el Consejo Nacional de Salud Mental. Se efectuaron nuevas estrategias basadas en el concepto de reducción del daño, ajustadas en respuesta a las necesidades del hospital (Figura 1). Se implementaron filtros sanitarios que permitieron detectar posibles casos de COVID-19 a partir de la presencia de sintomatología afín. Asimismo, se crearon algoritmos para el manejo y tratamiento de pacientes sospechosos o confirmados con COVID-19, que permitieron preparar al personal para una pronta y eficaz respuesta. También se llevaron a cabo pruebas de tamizaje entre el personal, lo que puso de manifiesto la existencia de casos confirmados de COVID-19 pero asintomáticos, con lo cual se identificó el posible origen de la infección nosocomial.

\section{Medidas de contención}

En este rubro se llevaron a cabo estrategias que permitieron mitigar la propagación del virus (Figura 2). De inicio, se reconvirtieron áreas especiales para la 


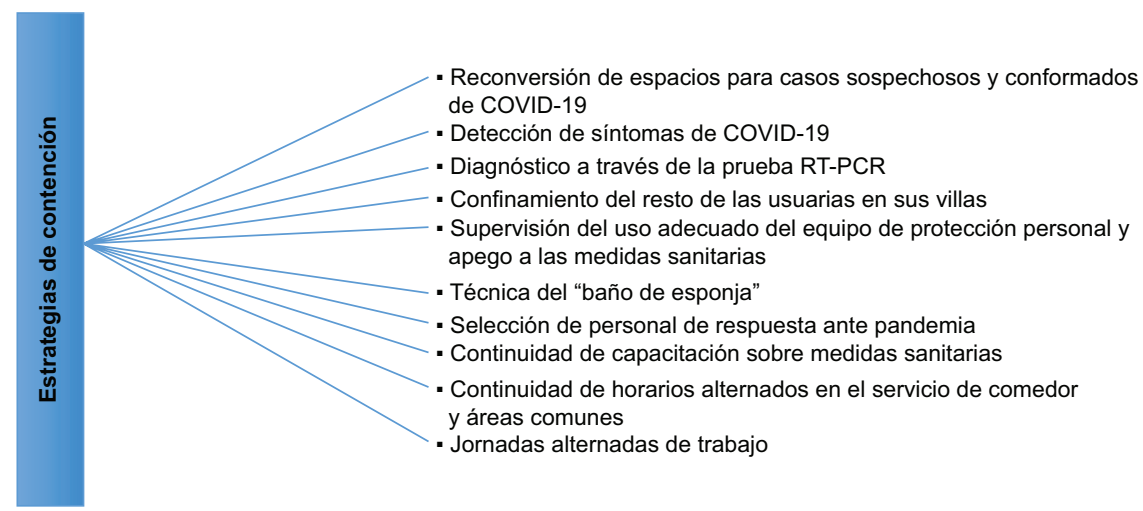

Figura 2. Estrategias de contención implementadas ante el brote de COVID-19 en un hospital psiquiátrico.

atención de casos sospechosos y confirmados de COVID-19, a las cuales solo tenía acceso el personal esencial para la atención de las usuarias. Dicho personal fue seleccionado considerando a quienes denotaran mayor fortaleza psicológica para contener el estrés y miedo, así como la deshidratación provocada por el uso de equipo de protección personal. Además, se supervisó el uso adecuado del equipo de protección personal y el apego a las medidas de sana distancia e higiene de manos en el resto del personal. Se establecieron horarios escalonados y jornadas alternadas de trabajo; específicamente, el área de enfermería estableció dos horarios dentro de un turno normal de servicio con el objetivo de no desgastar al personal e implementó el uso de "baños de esponja" para evitar la acumulación de gases y vapores que pudieran constituir una fuente de contagio. A la par, se indicó el confinamiento en villas del resto de las usuarias, acción clave para evitar el contagio nosocomial.

Ante esta situación, el área de psicología implementó terapia ocupacional y actividades encaminadas a evitar la exacerbación de sintomatología psiquiátrica y psicológica durante el confinamiento. También generó programas de atención dirigidos a los equipos de respuesta ante la pandemia. Por su parte, el área de trabajo social generó las estrategias para mantener la comunicación con los familiares de las usuarias. Finalmente, estas medidas ayudaron a aminorar el impacto general de la pandemia no solo en las usuarias, sino también en el personal que labora en el hospital psiquiátrico.

\section{Manejo y descripción de casos}

El 1 de julio de 2020 se reportaron cuatro casos considerados sospechosos de COVID-19 al presentar fiebre sin causa aparente; posteriormente se descartaron dos casos cuya causa respondía a infecciones bacterianas. A raíz de esto, se realizó la prueba para SARS-CoV-2 mediante reacción en cadena de la polimerasa en tiempo real (RT-PCR, confirmando así los primeros dos casos positivos, los cuales tuvieron fiebre). Adicionalmente, se realizó prueba RT-PCR a todas las mujeres con quienes las primeras pacientes positivas cohabitaban en la villa, aun cuando no presentaran síntomas. Durante los siguientes cinco días se acumuló un total de 19 mujeres con diagnóstico confirmado $(11.11 \%$ de la población total), quienes fueron puestas en aislamiento del 3 al 23 de julio del presente año. Si bien los protocolos de actuación indican periodos de aislamiento de 10 días, se decidió mantener dicha medida por 21 días para evitar al máximo un segundo grupo de contagios.

Posteriormente, se llevó a cabo la revisión sistemática de 19 expedientes para valorar los signos vitales (temperatura, tensión arterial, frecuencia cardiaca, frecuencia respiratoria, saturación de oxígeno) y se revisaron los estudios de laboratorio. En adición, se consideraron las comorbilidades que las usuarias tienen y la medicación que reciben de acuerdo con el diagnóstico psiquiátrico y médico previo al desarrollo de la infección por COVID-19 (Tabla 1).

Las usuarias positivas a COVID-19 fueron en su mayoría pacientes geriátricas, con edad de 43 a 95 años (media $=64$ años) y un tiempo de estancia en el hospital de siete meses a 61 años (media $=24$ años), a quienes se les proporcionó manejo conforme algoritmo para el tratamiento de pacientes sospechosos o confirmados con COVID-19, mencionado en el apartado de prevención. No se encontraron alteraciones importantes en las cifras 
Tabla 1. Diagnóstico psiquiátrico, comorbilidades, antecedentes farmacológicos y sintomatología COVID-19. Características clínicas de usuarias diagnosticadas con COVID-19

\begin{tabular}{|c|c|c|c|c|c|c|c|}
\hline Diagnóstico psiquiátrico & $\mathrm{n}$ & Comorbilidades & n & $\begin{array}{l}\text { Antecedentes } \\
\text { farmacológicos }\end{array}$ & $\mathrm{n}$ & $\begin{array}{l}\text { Sintomatología } \\
\text { COVID-19 }\end{array}$ & $\mathrm{n}$ \\
\hline Esquizofrenia paranoide & 2 & Epilepsia & 2 & Psicotrópicos & 19 & Artrialgias & 1 \\
\hline Esquizofrenia hebefrénica & 1 & Diabetes mellitus 2 & 1 & Antidiabéticos & 1 & Fiebre & 2 \\
\hline Esquizofrenia residual & 2 & Obesidad & 2 & Vasoprotectores & 1 & Febrícula & 1 \\
\hline Retraso mental profundo & 3 & TMC & 1 & Anticolinérgicos & 4 & Hipoxemia & 9 \\
\hline Retraso mental grave & 6 & Gonartrosis & 1 & Otro & 12 & & \\
\hline Retraso mental moderado & 3 & EPOC & 1 & & & & \\
\hline Trastorno bipolar & 1 & Gastritis crónica & 2 & & & & \\
\hline Demencia mixta & 1 & Linfoma & 1 & & & & \\
\hline
\end{tabular}

generales de temperatura (media $=36.2 \pm 0.32$ ), tensión arterial (media $=105 / 66 \pm 10,645)$, frecuencia cardiaca (media $=73.13 \pm 6.04)$ ni frecuencia respiratoria (media $=19.51 \pm$ : 0.46). Los estudios de laboratorio mostraron valores en rangos normales. Nueve casos presentaron una saturación arterial de oxígeno $<90$ por periodos alternados, que fue más aguda durante la tarde-noche. En dos casos se requirió $3 \mathrm{~L} /$ minuto de oxígeno mediante puntas nasales. En total, 13 casos presentaron sintomatología leve (específicamente fiebre, artralgias e hipoxemia) y seis s fueron asintomáticos. Los casos no mostraron alteraciones en el estado mental, la sintomatología psiquiátrica ni enfermedades de base.

\section{Conclusiones}

El contexto emergente de la pandemia por COVID19 ha significado el quiebre de muchos paradigmas en relación con el proceso de salud-enfermedad y, en consecuencia, se han tenido que adaptar numerosos lineamientos con el propósito de brindar una atención efectiva.

En ese sentido, en el caso que aquí se expone, el personal de salud que cursaba la enfermedad de forma asintomática propició un brote, aún con las medidas de prevención establecidas al inicio de la pandemia. Por consiguiente, una planeación que contemple estrategias integrales basadas en prevención, contención, mitigación y el pronto establecimiento de protocolos de actuación hará posible enfrentar los retos derivados de la presencia del COVID-19 o de cualquier enfermedad emergente.

Así, las estrategias generadas, así como los algoritmos para el manejo y tratamiento de pacientes diseñados por el equipo multidisciplinario establecen un precedente en el ámbito psiquiátrico con pacientes institucionalizados, que sirve como base para futuras experiencias emergentes.

La adecuada gestión de recursos humanos y los aportes brindados por el equipo multidisciplinario resultaron fundamentales en la implementación exitosa de las estrategias mencionadas.

Es importante mencionar que a pesar de las condiciones de enfermedades psiquiátricas aunadas al hecho de la presencia de comorbilidades en la población descrita, el curso de la infección por COVID-19 no generó afectaciones sustanciales a nivel sistémico. Contrario a lo reportado previamente, no se observaron alteraciones en la enfermedad de base ni en la sintomatología provocada por la infección por COVID-19, por lo que no se requirieron medidas de atención de urgencia u hospitalización. Este hecho merece ser estudiado a profundidad, ya que denota que algún factor protector permite que los pacientes con estas características cursen sin complicaciones esta y otras enfermedades.

Finalmente, COVID-19 se presentó como una ventana de oportunidad para innovar y mejorar los procesos que permiten reducir el índice de infecciones, entendiendo que las medidas descritas se convertirán en medidas permanentes pues se comprobó su 
efectividad al minimizar el daño provocado por la pandemia.

\section{Conflicto de intereses}

No existe ningún conflicto de intereses.

\section{Financiamiento}

Los autores no recibieron financiamiento para esta investigación.

\section{Responsabilidades éticas}

Protección de personas y animales. Los autores declaran que para esta investigación no realizaron experimentos en seres humanos ni en animales.

Confidencialidad de los datos. Los autores declaran que siguieron los protocolos de su centro de trabajo sobre la publicación de datos de pacientes.

Derecho a la privacidad y consentimiento informado. Los autores declaran que en este artículo no aparecen datos de pacientes.

\section{Bibliografía}

1. Cennimo D, Olsen K. Coronavirus disease 2019 (COVID-19): practice essentials, background, route of transmission. Medscape. 2021.

2. Yao $H$, Chen $J-H, X u Y-F$. Patients with mental health disorders in the COVID-19 epidemic. Lancet Psychiatry. 2020;7:e21.

3. Niu S, Tian S, Lou J, Kang X, Zhang L, Lian H, et al. Clinical characteristics of older patients infected with COVID-19: a descriptive study. Arch Gerontol Geriatr. 2020;89:104058.

4. Richardson S, Hirsch JS, Narasimhan M, Crawford JM, McGinn T, Davidson $\mathrm{KW}$, et al. Presenting characteristics, comorbidities, and outcomes among 5700 patients hospitalized with COVID-19 in the New York City area. JAMA. 2020;323:2052-2059.

5. Huang I, Lim MA, Pranata R. Diabetes mellitus is associated with increased mortality and severity of disease in COVID-19 pneumonia - a systematic review, meta-analysis, and meta-regression. Diabetes Metab Syndr. 2020;14:395-403.

6. Yang J, Zheng Y, Gou X, Pu K, Chen Z, Guo Q, et al. Prevalence of comorbidities and its effects in patients infected with SARS-CoV-2: a systematic review and meta-analysis. Int J Infect Dis. 2020;94:91-95.

7. Ji H, Liu L, Huang T, Zhu Y. Nosocomial infections in psychiatrich hospitals during the COVID-19 outbreak. Eur J Psychiat. 2020;34:177-179.

8. Chen J, Xiong M, He Z, Shi W, Yue Y, He M. The enclosed ward management strategies in psychiatric hospitals during COVID-19 outbreak. Global Health. 2020;16:53.

9. Hsu S-T, Chou L-S, Chou FH-C, Hsieh K-Y, Chen C-L, Lu WC, et al. Challenge and strategies of infection control in psychiatric hospitals during biological disasters-From SARS to COVID-19 in Taiwan. Asian J Psychiatr. 2020;54:102270.

10. Gaspard P, Mosnier A, Gunther D, Lochert C, Larocca S, Minery P, et al. Influenza outbreaks management in a French psychiatric hospital from 2004 to 2012. Gen Hosp Psychiatry. 2014;36:46-52.

11. Lineamientos generales para la mitigación y prevención de COVID-19 en os hospitales psiquiátricos [En línea]. México: Gobierno de México; 2020.

12. Son 3 muertos por brote de Covid-19 en siquiátrico del Edomex: $\mathrm{CNDH}$. La Jornada. 2020 Jun 20. 